\title{
Raiva em herbívoros no estado do Maranhão: um estudo retrospectivo
}

\section{Rabies in herbivorous in the state of Maranhão: a retrospective study}

\author{
Daniela Rios Póvoas, ${ }^{*}$ Nancyleni Pinto Chaves, ${ }^{* *}$ Danilo Cutrim Bezerra, ${ }^{* * *}$ Viramy Marques de Almeida, ${ }^{*}$ \\ Lauro de Queiroz Saraiva*
}

\section{Resumo}

O objetivo do trabalho foi determinar a frequência relativa de herbívoros positivos para a raiva no estado do Maranhão, utilizando dados da Agência de Defesa Agropecuária do Estado, coletados no período de 2006 a 2010. Durante esse período, foram examinadas 193 amostras provenientes de herbívoros. Das amostras avaliadas, 35,75\% (69/193) foram positivas para o vírus rábico. Houve diferença estatística significativa $(P<0,05)$ na proporção de herbívoros positivos por período avaliado. Os resultados indicam que no período avaliado, a raiva no Estado do Maranhão apresentou frequência relativa alta. Esses achados evidenciam a necessidade da realização de diagnóstico sistemático e monitoramento dos rebanhos, além da implantação de medidas de controle e profilaxia e vacinações.

Palavras-chave: raiva, frequência, diagnóstico laboratorial, Maranhão.

\begin{abstract}
The objective of this study was to determine the relative frequency of herbivores positive for rabies in the state of Maranhão, using data from the Defense Agency of the State Agricultural, collected in the period 2006 to 2010. During this period, 193 samples from herbivores were examined. Of the samples, $35.77 \%(69 / 193)$ were positive for the rabies virus. There was a statistically significant difference $(P<0.05)$ in the proportion of herbivores by positive evaluation period. The results indicate that during this period, the rabies in the state of Maranhão showed relatively high frequency. These findings highlight the need to carry out systematic diagnosis and monitoring of herds, besides the implementation of control measures and prophylaxis and vaccinations.
\end{abstract}

Keywords: rabies, frequency, laboratory diagnosis, Maranhão.

\section{Introdução}

A raiva é considerada uma das zoonoses de maior importância em Saúde Pública, pela sua evolução invariavelmente fatal e pelo custo social e econômico que ocasiona. A doença é causada pelo vírus da raiva, pertencente ao gênero Lyssavirus, família Rhabdoviridae, ordem Mononegavirales (Lima et al. 2005, Gomes et al. 2007, Brasil, 2009).

A enfermidade é mantida e perpetuada na natureza por várias espécies animais, denominadas reservatórios, como carnívoros domésticos e silvestres ou morcegos de diferentes hábitos alimentares (Rupprecht et al. 2002). Tradicionalmente, o ciclo de transmissão da raiva pode ser dividido em dois ciclos interrelacionados: o ciclo urbano e o ciclo silvestre (Acha e Szyfres 2003, Kobayashi et al. 2006). Entretanto, segundo Rupprecht et al. (2001), essa descrição muito simplificada do ciclo silvestre e urbano não permite esclarecer a real dinâmica da doença.

No Brasil, o ciclo silvestre da raiva é mantido, preferencialmente, por morcegos hematófagos transmissores da doença aos bovinos, outros mamíferos silvestres e ocasionalmente ao homem, e o ciclo urbano normalmente é mantido pelo cão doméstico (Acha e Szyfres, 2003, Kobayashi et al., 2006).

Surtos de raiva em bovinos são descritos no Brasil desde 1911 (Carini, 1911) e, a partir de 1966 implantou-se o Plano de Combate à Raiva dos Herbívoros, atualmente, denominado Programa Nacional de Controle da Raiva dos Herbívoros e outras Encefalopatias (PNCRH), que tem como objetivo principal diminuir a prevalência da doença nos herbívoros domésticos (Brasil, 2009).

Mesmo com a implantação deste Programa, a raiva ainda ocorre de maneira endêmica no Brasil. Estima-se que a raiva bovina na América Latina cause prejuízos anuais de centenas de milhões de dólares, provocados pela morte de milhares de cabeças, além dos gastos indiretos que podem ocorrer com a vacinação de milhões de bovinos e inúmeros tratamentos pósexposição (sorovacinação) de pessoas que mantiveram contato com animais suspeitos (Brasil, 2009).

Em herbívoros, a raiva pode apresentar variações no quadro clínico, e muitos dos sinais podem estar presentes em outras

\footnotetext{
* Fiscais Estaduais Agropecuários, Agência Estadual de Defesa Agropecuária do Maranhão, AGED-MA, São Luís - MA.

${ }^{* *}$ Doutoranda em Biotecnologia, Rede Nordeste de Biotecnologia, RENORBIO, São Luís - MA. Autor para correspondência: nancylenichaves@hotmail.com.

*** Mestre em Ciências Veterinárias, Universidade Estadual do Maranhão, Escola de Medicina Veterinária, São Luís - MA.
} 
doenças que causam distúrbios neurológicos. Assim, o exame clínico não deve ser utilizado como único critério para o diagnóstico da enfermidade (Mori e Lemos, 1998).

A Organização Mundial de Saúde preconiza para diagnóstico da raiva as técnicas de Imunofluorescência Direta (IFD) e prova biológica (inoculação intracerebral em camundongos - IIC (WHO, 1996).

Para o combate à raiva dos herbívoros, ações conjuntas devem ser implementadas, como: controle de morcegos hematófagos, vacinação sistemática de herbívoros, e educação em saúde. Em áreas epizoóticas, a vacinação deve ser semestral (Kotait et al., 1998).

O estado do Maranhão possui uma área territorial de $331.983,293 \mathrm{Km}^{2}$, localizado a Noroeste da Região Nordeste. Limita-se ao Norte com o Oceano Atlântico, Sul e Sudoeste ao estado do Tocantins, Leste e Sudeste com o estado do Piauí e ao Oeste com o estado do Pará, possui uma população estimada em 6.103.327 habitantes distribuída em 217 municípios (IBGE, 2011). O estado conta com 85.891 estabelecimentos pecuários criadores de herbívoros, com efetivo de 12.820.421 animais (Maranhão, 2010). O Serviço de Defesa Agropecuária do Estado do Maranhão (AGED-MA) possui 70 Unidades Locais de Sanidade Animal e Vegetal (ULSAV), 18 gerências regionais e uma unidade central.

Nesse contexto potencialmente crítico, em que infecção e doença representam praticamente o mesmo risco na cadeia epidemiológica, este trabalho foi estruturado com o objetivo de determinar a frequência de herbívoros positivos ao vírus rábico, utilizando dados do Serviço de Defesa Agropecuária do Estado do Maranhão, no período de 2006 a 2010.

\section{Material e métodos}

No presente trabalho, foram utilizados os condensados estaduais dos informes mensais, FORM-INs e FORMCOMs sobre a ocorrência e o diagnóstico clínico e laboratorial da raiva. O período do estudo foi de janeiro de 2006 a dezembro de 2010.

O diagnóstico da enfermidade foi realizado pelas técnicas de Imunofluorescência Direta (IFD) e inoculação intracerebral em camundongos (ICC), no Laboratório de Raiva da Universidade Estadual do Maranhão (UEMA).

Foram calculadas as frequências relativas de animais positivos por ano e espécie animal acometida. Para a comparação dos dados de frequência por ano de observação utilizou-se estatística por meio do teste Quiquadrado de independência. O nível de significância utilizado na decisão dos testes estatísticos foi de $5 \%$ $(0,05)$ e obtiveram-se intervalos com confiabilidade de $95 \%$. O programa utilizado para a obtenção da análise foi o Eplnfo 3.43 versão 2007.

\section{Resultados e discussão}

Foram avaliados 193 laudos laboratoriais de amostras provenientes de herbívoros, no período de janeiro de 2006 a dezembro de 2010. Deste total, 174 amostras eram de bovinos, nove de equinos, quatro de asininos, três de ovinos, duas de caprinos e uma de muar. Os resultados laboratoriais por período avaliado e espécie animal encontram-se discriminados na Tabela 1.

Tabela 1: Resultados dos testes laboratoriais para raiva em 193 amostras provenientes de herbívoros, no período de 20062010

\begin{tabular}{|c|c|c|c|c|c|c|}
\hline \multicolumn{7}{|c|}{ Ano Base: 2006} \\
\hline \multirow{2}{*}{ Espécie Animal } & \multicolumn{2}{|c|}{ Positivos } & \multicolumn{2}{|c|}{ Negativos } & \multicolumn{2}{|c|}{ Total } \\
\hline & $\mathrm{n}$ & $\%$ & $\mathrm{~N}$ & $\%$ & $\mathrm{~N}$ & $\%$ \\
\hline Bovina & 18 & 37,50 & 25 & 52,08 & 43 & 89,58 \\
\hline Asinina & 1 & 2,08 & 0 & 0,00 & 1 & 2,08 \\
\hline Ovina & 0 & 0,00 & 2 & 4,17 & 2 & 4,16 \\
\hline Equina & 0 & 0,00 & 2 & 4,17 & 2 & 4,16 \\
\hline Total & 19 & 39,58 & 29 & 60,42 & 48 & 100,00 \\
\hline \multicolumn{7}{|c|}{ Ano Base: 2007} \\
\hline \multirow[t]{2}{*}{ Espécie Animal } & \multicolumn{2}{|c|}{ Positivos } & \multicolumn{2}{|c|}{ Negativos } & \multicolumn{2}{|c|}{ Total } \\
\hline & $\mathrm{n}$ & $\%$ & $\mathrm{n}$ & $\%$ & $\mathrm{~N}$ & $\%$ \\
\hline Bovina & 17 & 47,22 & 15 & 41,66 & 32 & 88,88 \\
\hline Equina & 1 & 2,78 & 0 & 0,00 & 1 & 2,78 \\
\hline Caprina & 1 & 2,78 & 1 & 2,78 & 2 & 2,57 \\
\hline Ovina & 1 & 2,78 & 0 & 0,00 & 1 & 2,78 \\
\hline Total & 20 & 55,56 & 16 & 44,44 & 36 & 100,00 \\
\hline \multicolumn{7}{|c|}{ Ano Base: 2008} \\
\hline \multirow[t]{2}{*}{ Espécie Animal } & \multicolumn{2}{|c|}{ Positivos } & \multicolumn{2}{|c|}{ Negativos } & \multicolumn{2}{|c|}{ Total } \\
\hline & $\mathrm{n}$ & $\%$ & $\mathrm{n}$ & $\%$ & $\mathrm{~N}$ & $\%$ \\
\hline Bovina & 11 & 26,82 & 25 & 60,98 & 36 & 87,80 \\
\hline Equina & 2 & 4,88 & 1 & 2,43 & 3 & 7,31 \\
\hline Asinino & 0 & 0,00 & 2 & 4,88 & 2 & 4,88 \\
\hline Total & 13 & 31,70 & 28 & 68,30 & 41 & 100,00 \\
\hline \multicolumn{7}{|c|}{ Ano Base: 2009} \\
\hline \multirow[t]{2}{*}{ Espécie Animal } & \multicolumn{2}{|c|}{ Positivos } & \multicolumn{2}{|c|}{ Negativos } & \multicolumn{2}{|c|}{ Total } \\
\hline & $\mathrm{n}$ & $\%$ & $\mathrm{n}$ & $\%$ & $\mathrm{~N}$ & $\%$ \\
\hline Bovina & 7 & 25,92 & 20 & 74,08 & 27 & 100,00 \\
\hline \multicolumn{7}{|c|}{ Ano Base: 2010} \\
\hline \multirow[t]{2}{*}{ Espécie Animal } & \multicolumn{2}{|c|}{ Positivos } & \multicolumn{2}{|c|}{ Negativos } & \multicolumn{2}{|c|}{ Total } \\
\hline & $\mathrm{n}$ & $\%$ & $\mathrm{n}$ & $\%$ & $\mathrm{~N}$ & $\%$ \\
\hline Bovina & 10 & 24,40 & 26 & 63,41 & 36 & 87,81 \\
\hline Equina & 0 & 0,00 & 3 & 7,31 & 3 & 7,31 \\
\hline Asinino & 0 & 0,00 & 1 & 2,44 & 1 & 2,44 \\
\hline Muar & 0 & 0,00 & 1 & 2,44 & 1 & 2,44 \\
\hline Total & 10 & 24,40 & 31 & 76,00 & 41 & 100,00 \\
\hline
\end{tabular}

No estudo foi observada frequência relativa de 35,75\% (69/193) de herbívoros positivos com 97,80\% (91/93) dos casos localizados na zona rural. No Brasil, a raiva dos herbívoros é considerada endêmica e em graus diferenciados, de acordo com a região do País (Brasil, 2005).

Com relação ao número de casos de raiva no período estudado, em vista ao tamanho do rebanho maranhense, os valores podem estar 
muito aquém da realidade. De acordo com Kotait et al. (1998), para cada caso de raiva bovina notificado, cerca de 10 casos não o são. Esta afirmação corrobora com a situação atual da raiva bovina no Brasil, onde o elevado número de subnotificações compromete o programa de controle e erradicação desta enfermidade (Brasil, 2005).

Das espécies avaliadas, o maior percentual de casos positivos foi para a espécie bovina com valores de frequências relativas de $39,58 \%, 55,56 \%, 31,70 \%, 25,92 \%$ e $24,42 \%$ para os anos de 2006, 2007, 2008, 2009 e 2010, respectivamente.

Os relatos de raiva em pequenos ruminantes são escassos. No presente estudo foram evidenciados caprinos e ovinos positivos. Entretanto, com casuística inferior à raiva em bovinos. Situação semelhante foi encontrada para equídeos (equinos e asininos). Outros trabalhos também evidenciam a raiva em pequenos ruminantes e equinos, entretanto, com pequeno número de casos (Guedes et al. 2007; Nantes e Zappa, 2008, Rissi et al. 2008; Silva et al., 2010).

A raiva dos herbívoros, principalmente a raiva dos bovinos, transmitida pelo morcego hematófago Desmodus rotundus, representa uma importante limitação ao desenvolvimento da pecuária na América Latina. A grande repercussão da raiva na pecuária mundial são as perdas calculadas de aproximadamente 850 mil cabeças que equivalem a aproximadamente 17 milhões de dólares (Lima et al. 2005).

Os herbívoros são hospedeiros acidentais do vírus rábico e, apesar de integrarem a cadeia epidemiológica, atuam apenas como sentinelas e hospedeiros terminais da doença. Assim, a probabilidade de transmissão para outros animais é baixa (Rupprecht et al. 2002).

Para o período avaliado foram identificadas frequências relativas de $39,85 \%$ (2006), 55,56\% (2007), 31,70\% (2008), 25,92\% (2009) e $24,40 \%$ (2010), com diferença estatística significativa $(\mathrm{P}<0.05)$ por período (ano) avaliado. Observa-se redução significativa dos casos de raiva no período de 2008 a 2010. Isso provavelmente ocorreu em função de um grande número de casos diagnosticados e divulgados entre 2005 e 2007, que levou a vacinação dos rebanhos no estado e o controle da enfermidade. Equipes de vigilância epidemiológica da AGED-MA foram formadas para a realização de fiscalização ativa nas áreas afetadas. Associado a isto, a captura e combate do morcego hematófago foi intensificado no mesmo período.

Os principais fatores que podem ter contribuído para a ocorrência de raiva nos herbívoros domésticos no estado do Maranhão foram: aumento da oferta de alimento, representado pelo significativo crescimento dos rebanhos, ocupação desordenada, caracterizada por macromodificações ambientais, como desmatamento, construção de rodovias e hidroelétricas, que alteraram o ambiente em que os morcegos vivem, obrigando-os a procurar novas áreas e outras fontes de alimentação; oferta de abrigos artificiais, representados pelas construções, como túneis, cisternas, casas abandonadas, bueiros, fornos de carvão desativados.

Neste levantamento foi possível a identificação da raiva ao longo de todos os meses de observação, não havendo maior concentração de casos para uma determinada época do ano, corroborando com Lima et al. (2005). Entretanto, em levantamentos realizados na Região Central do Rio Grande do Sul, foram identificados maior número de casos na primavera e verão (Pereira et al., 2011) evidenciando que existe tendência de sazonalidade da raiva com o maior número de casos ocorrendo em determinada época do ano.

O período de acasalamento de morcegos ocorre na primavera. Estudos indicam que neste período haveria aumento da transmissão do vírus entre as colônias. O pico de surtos ocorreria no outono em função do período de incubação da doença nos morcegos e nos herbívoros agredidos (Mori e Lemos, 1998).

Para Teixeira et al. (2008), a raiva é uma enfermidade cíclica, reaparecendo com periodicidade de aproximadamente 7 anos. Isso se deve aparentemente ao maior número de morcegos infectados nos picos da doença nos herbívoros, ocorrendo período de declínio, para repovoamento e reinfecção das colônias de morcegos, já que o crescimento das mesmas é lento.

Com a avaliação de FORM-INs e FORM-COMs foi possível a identificação da forma paralítica da raiva. Os sinais clínicos especificados caracterizaram-se por apatia ou inquietação, mudança de comportamento, incoordenação motora, seguida de paresia e paralisia inicialmente dos membros pélvicos, decúbito, movimentos de pedalagem, opistótono e morte.

Como o estado de Maranhão possui o segundo maior rebanho bovino da região Nordeste, uma extensa área territorial e biomas diferenciados, o incremento de medidas profiláticas mais efetivas contra a raiva deve ser priorizada. Segundo Taddei (1991), a velocidade de migração da raiva pode alcançar valores variando de 10 a $200 \mathrm{~km} / \mathrm{ano}$. Por outro lado, devem ser avaliadas as condições ambientais favoráveis à manutenção do agente etiológico e disseminação deste pelos morcegos que encontram alimento em abundância, principalmente em criações de bovinos.

Com base nos resultados obtidos, pode-se concluir que: a frequência relativa de herbívoros positivos $(35,75 \%)$ para raiva no estado do Maranhão, no período avaliado, foi elevada; a espécie bovina foi a mais acometida em todos os períodos de avaliação; houve redução significativa da raiva em herbívoros no período de 2008 a 2010.

Considerando as condições econômicas da produção de bovinos do estado, bem como a ausência de planejamento amostral adequado, sugere-se que esforços sejam concentrados na intensificação da vacinação, captura de morcegos e educação em saúde.

\section{Agradecimento}

À Agência Estadual de Defesa Agropecuária do Maranhão (AGED-MA) pela concessão dos dados. 


\section{Referências}

ACHA, P. N.; SZYFRES, B. Zoonosis y enfermedades transmisibles comunes al hombre y a los animales. 3. ed., Washington, DC, Organización Panamericana de la Salud, v. 2, 2003, 425 p.

BRASIL. Controle da raiva dos herbívoros. Departamento de Saúde Animal, Secretaria de Defesa Agropecuária, Ministério da Agricultura, Pecuária e Abastecimento, Brasília, DF. 2005, 104 p.

BRASIL. Raiva humana, Brasil, 1986-2009. Coordenação de Vigilância das Doenças Transmissíveis por Vetores e Antropozoonoses, Coordenação Geral de Doenças Transmissíveis, Secretaria de Vigilância em Saúde, Ministério da Saúde, 2009. Acesso em: 25 de janeiro de 2011. http://portal. saude.gov.br.

CARINI, A. Sur une grande épizootic de rage. Annales de l'Institut Pasteur, v. 25, p. 843-846, 1911.

GOMES, M.N.; MONTEIRO, A.M.V.; NOGUEIRA, V.S.; GONÇALVES, C.A. Áreas propícias para o ataque de morcegos hematófagos Desmodus rotundu em bovinos na região de São João da Boa Vista, estado de São Paulo. Pesquisa Veterinária Brasileira, v. 27, p. 307-313, 2007.

GUEDES, K.M.R.; RIET-CORREA, F.; DANTAS, A.F.M.; SIMÕES, S.V.D.; NETO, E.G.M.; NOBRE, V.M.T.; MEDEIROS, R.M.T. Doenças do sistema nervoso central em caprinos e ovinos no semiárido. Pesquisa Veterinária Brasileira, v. 27, n. 1, p. 29-38, 2007.

IBGE. Instituto Brasileiro de Geografia e Estatística, 2011. Disponível em:<http://www. ibge.gov.com.br. Acesso em: 15 de janeiro de 2011.

KOBAYASHI, Y.; OGAWA, A.; SATO, G.; SATO, T., ITOU, T.; SAMARA ,S.I.; CARVALHO, A.A.B.; NOCITI, D.P.; ITO, F.H.; SAKAI, T. Geographical distribution of vampire bat-related cattle rabies in Brazil. Journal of Veterinary Medical Science, v. 68, n. 10 p. 1097-1100, 2006.

KOTAIT, I.; GONÇALVES, C.A.; PERES, N.F.; SOUZA, M.C.A.M.; TARGUETA, M.C. Controle da raiva dos herbívoros. Manual Técnico do Instituto Pasteur, São Paulo, v.1, p. 5-11, 1988.

LIMA, E.F.; RIET-CORREA, F.; CASTRO, R.S.; GOMES, A.A.B.; LIMA, F.S. Sinais clínicos, distribuição das lesões no sistema nervoso central e epidemiologia da raiva em herbívoros na região Nordeste do Brasil. Pesquisa Veterinária Brasileira, v. 25, n. 4, p. 250-264, 2005.
MARANHÃO. Agência Estadual de Defesa Agropecuária do Maranhão (AGED-MA). Setor de Epidemiologia e Estatística. Efetivo rebanho do Estado do Maranhão, 2010.

MORI, A.E.; LEMOS, R.A.A. Raiva, p. 47-58, 1998. In: Lemos R.A.A. (Ed.), Principais enfermidades de bovinos de corte do Mato Grosso do Sul: reconhecimento e diagnóstico. Universidade Federal do Mato Grosso do Sul, Campo Grande, MS.

NANTES, J.H.; ZAPPA, V. Raiva equina - relato de caso. Revista Científica Eletrônica de Medicina Veterinária, n. 11, 2008.

PEREIRA, C.M.; SALLIS, E. S.V.; GRECCO, F.B.; RAFFI, M.B.; SOARES, M.P.; SCHILD, A.L. Raiva em bovinos na Região Sul do Rio Grande do Sul: epidemiologia e diagnóstico imunohistoquímico. Pesquisa Veterinária Brasileira, v. 31, n. 4, p. 331335, 2011.

RISSI, D.R.; PIEREZAN, F.; KOMMERS, G.D.; BARROS, C.S.L. Ocorrência de raiva em ovinos no Rio Grande do Sul. Pesquisa Veterinária Brasileira, v. 28, n.10, p. 495-500, 2008.

RUPPRECHT, C.E.; STOHR, K.; MEREDITH, C. Rabies. In: WILLIANS, E. S.; BARKER, I. K. Infections diseases of wild mammals. lowa: lowa State University Press, p. 3-36, 2001.

RUPPRECHT, C.E.; HANLON, C.A.; HEMACHUDHA, T. Rabies re-examined. Lancet Infectious Diseases, v. 2, n. 6, p. 327-343, 2002.

SILVA, M.L.C.R. A.; RIET-CORREA, F.; GALIZA, G.J.N.; AZEVEDO, S.S.; AFONSO, J. A.B.; GOMES, A. A.B. Distribuição do vírus rábico no sistema nervoso central em ruminantes naturalmente infectados. Pesquisa Veterinária Brasileira, v. 30, n. 11, p. 940-944, 2010.

TADDEI, A.V.; GONÇALVES, C.A.; PEDRO, W.A. Distribuição do morcego vampiro Desmodus rotundus no estado de São Paulo e a raiva dos animais domésticos. Campinas: Coordenadoria de Assistência Técnica Integral, 1991. 107 p.

TEIXEIRA, T.F.; HOLZ, C.L.; CAIXETA, S.P.M.B.; DEZEN, D.; CIBULSKI, S.P.; SILVA, J.R.; ROSA, J.C.A.; SCHMIDT, E.; FERREIRA, J.C.; BATISTA, H.B.C.R.; CALDAS, E.; FRANCO, A.C.; ROEHE, P.M. Diagnóstico de raiva no Rio Grande do Sul, Brasil, de 1985 a 2007. Pesquisa Veterinária Brasileira, v. 28, p. 515-520, 2008.

WHO. WORD SURVEY OF RABIES, 32 - For the year, 1996 Diseases surveillance and control. WHO/EMC/ZDI/98.4, 1996. 